\title{
Ambulation and independence among Veterans with nontraumatic bilateral lower-limb loss
}

\author{
Sherene E. Sharath, MPH; ${ }^{1}$ Helene Henson, MD; ${ }^{2-3}$ Stacy Flynn, PT, CWS; ${ }^{2}$ George Pisimisis, MD; ${ }^{4}$ Panos \\ Kougias, MD, MS; ${ }^{4}$ Neal R. Barshes, MD, MPH $^{4 *}$ \\ ${ }^{1}$ Health Services Research \& Development, Michael E. DeBakey Department of Veterans Affairs (VA) Medical Center, \\ Houston, TX; ${ }^{2}$ Amputee System of Care, Michael E. DeBakey VA Medical Center, Houston, TX; ${ }^{3}$ Department of Physi- \\ cal Medicine and Rehabilitation, Baylor College of Medicine, Houston, TX; ${ }^{4}$ Division of Vascular Surgery and Endo- \\ vascular Therapy, Michael E. DeBakey Department of Surgery, Baylor College of Medicine/Michael E. DeBakey VA \\ Medical Center, Houston, TX
}

\begin{abstract}
In describing functional outcomes and independent living in a cohort of bilateral major amputees, we sought to provide current estimates of function and independence after a second major amputation in an elderly Veteran population with peripheral arterial disease and/or diabetes. After retrospectively reviewing and excluding the electronic health records of those failing to meet the inclusion criteria, we identified 40 patients with a history of unilateral major amputation who underwent a second major amputation during the defined study period. Of these, $43 \%$ (17) were bilateral transfemoral amputations (TFAs); bilateral transtibial amputations (TTAs) and TFA-TTA accounted for the rest (33\% and 25\%, respectively). Of the $19(48 \%)$ patients who were ambulatory prior to bilateral amputation, only $2(11 \%)$ remained ambulatory after the second amputation, while $17(89 \%)$ patients lost ambulatory capabilities. Compared with those who were $<=65 \mathrm{yr}$, those between 66 and $79 \mathrm{yr}$ were $18 \%$ less likely to ambulate precontralateral amputation $(p=0.03)$. All patients with bilateral TFA were nonambulatory. Independence postcontralateral amputation decreased from $88 \%$ (35) to $53 \%$ (21). When data were available (58\%), pre and post Functional Independence Measure scores showed a decrease in $74 \%$ of patients, while $22 \%$ showed an increase. In conclusion, bilateral lower-limb amputation among dysvascular Veterans is highly associated with a loss of ambulation.
\end{abstract}

Key words: activities of daily living, ambulation, amputations, diabetes, Functional Independence Measure, functional outcomes, independence, limb loss, transfemoral amputations, transtibial amputations.

\section{INTRODUCTION}

A previous history of contralateral lower-limb limb loss has been identified as a risk factor for poor outcomes following revascularization and limb preservation efforts [1]. Although these poorer expected outcomes may make some clinicians more cautious in entertaining the idea of limb preservation efforts in patients who have a history of unilateral amputation, the alternative - limb loss in the remaining lower limb - is no more appealing.

Among ambulatory patients with critical limb ischemia and without a history of prior major amputation, ambulation rates following infrainguinal bypass are 97 percent [2$3]$ but are only 20 percent among those with such a history [4]. Previous reports suggest a comparable disadvantage

Abbreviations: ADL $=$ activity of daily living, $\mathrm{CAD}=$ coronary artery disease, ESRD = end-stage renal disease, FIM = Functional Independence Measure, $\mathrm{PAD}=$ peripheral arterial disease, TFA $=$ transfemoral amputation, TTA $=$ transtibial amputation, VA = Department of Veterans Affairs.

*Address all correspondence to Neal R. Barshes, MD, MPH; Division of Vascular Surgery and Endovascular Therapy, Michael E. DeBakey Department of Surgery, Baylor College of Medicine/Michael E. DeBakey VA Medical Center, 2002 Holcombe Blvd (OCL 112), Houston, TX 77030; 713-7911414, ext 26625. Email: nbarshes@bcm.tmc.edu http://dx.doi.org/10.1682/JRRD.2014.07.0176 
following major amputation: whereas about 55 percent of those undergoing nontraumatic major lower-limb amputation remain ambulatory [5] only about 38 percent of those with a prior history of contralateral limb loss remain ambulatory following loss of the second lower limb [6]. The few studies published on function after bilateral major amputations have not focused on the elderly population with peripheral arterial disease (PAD) and/or diabetes typically encountered by the vascular surgeon. Nonetheless, these studies suggest that 60 percent lose ambulatory ability and 65 percent lose their ability to live independently [6-7].

Patients who have undergone major amputation have many challenges. First, the contralateral (intact) foot has a 9-fold higher risk of ulceration and eventual amputation (as high as $25 \%$ over $10 \mathrm{yr}$ ) compared with patients with similar risk factors but without a history of major amputation [8-9]. Additionally, rates of successful limb salvage following revascularization are lower among patients with a history of contralateral major amputation [1]. The poorer clinical outcomes experienced by patients with a history of major amputation are probably due to a higher prevalence of risk factors such as end-stage renal disease (ESRD) [1] and neuropathy but perhaps are also due to the elevated plantar pressures that occur when ambulating with a limb prosthesis [10].

With this in mind, we undertook this descriptive study to present estimates of functional outcomes (ambulation and independent living) for nontraumatic, bilateral, major lower-limb amputations in an elderly Veteran population with PAD and/or diabetes mellitus. The objective was to provide estimates of patient-centered outcomes that would be useful for shared decision-making in the clinical setting $[5,11]$.

\section{METHODS}

\section{Study Subjects}

Patients with a previous history of major amputation, who subsequently underwent major amputation of the contralateral (intact) lower limb at the Michael E. DeBakey Department of Veterans Affairs (VA) Medical Center between June 1998 and December 2012, were included in this study $(n=41)$. Patients were retrospectively identified using a prospectively maintained registry of major operative cases at our institution. Major amputation was defined as any amputation above the level of the malleoli of the ankle, including transtibial amputations (TTAs), through-knee amputations, or transfemoral amputations (TFAs). Hip disarticulations were excluded from the study $(n=1)$.

Data on baseline demographic characteristics and comorbidities were collected from the electronic medical records. This review included an assessment of the presence of comorbidities such as diabetes mellitus, ESRD, and coronary artery disease (CAD). PAD was defined as an ankle-brachial index of $<0.9$ or any history of previous lower-limb revascularization.

\section{Measures}

The primary functional endpoints were ambulation and independent living. Baseline (up to $1 \mathrm{yr}$ prior to contralateral amputation) and postoperative ambulation was defined as the patient-reported primary means of locomotor ability (i.e., ambulation with or without a limb prosthesis and/or assistive device vs use of a wheelchair as primary mobility aid). All patients received an evaluation and subsequent therapy by a team composed of a physical medicine and rehabilitation physician and physical therapists. Mobility was categorized as ambulatory versus bedridden/nonambulatory as described in physician notes. Ambulation was further distinguished between household and community. Patient-reported ambulatory capabilities and disposition were identified through physical medicine and history and physical as well as prosthesis notes. Reports with discrepant information were evaluated, and the report with more depth of information and therefore higher quality was used.

The Functional Independence Measure (FIM) score as assessed by a certified physical therapist or physical medicine and rehabilitation physician was used as a measure of overall baseline and postoperative function. This scale is a validated assessment of two components of function: (1) mobility, including locomotion, community mobility, and transfers; and (2) ability to perform activities of daily living (ADLs), including eating, grooming, dressing, and bathing. The FIM has been used and validated across a variety of patients and settings, and a large study conducted in an inpatient rehabilitation setting demonstrated that this scale has high discriminative ability and internal consistency [12-13]. Additionally, data on KLevels, as defined by Medicare and assessed by the physical therapists, were collected when available. Where level 0 indicates an absence of ability or potential to ambulate or transfer safely, levels 1 to 4 range from household 
ambulatory ability to those with the potential or ability to perform the activities of an energetic adult [14].

Independence was defined as the ability to live in the community without long-term support for ADLs, categorized based on living situation at last known follow-up: residing at home without professional nursing support, residing in skilled nursing facility or at home with professional long-term home nursing support, or residing in a nursing home.

\section{Analysis}

Nonparameteric measures and tests were used for all descriptive statistics and basic comparisons. Specifically, a two-sided Fisher exact test was used to identify whether significant differences existed between groups (i.e., postoperative ambulation and independence vs comorbidities). Outcomes of interest (i.e., postoperative ambulation, postoperative independent living) were modeled against comorbidities and risk factors (e.g., diabetes, ESRD, PAD, preoperative ambulation, preoperative independent living) using univariate and multivariate logistic regressions. A pvalue of $<0.05$ was considered statistically significant. Intercooled Stata (version 12.0, StataCorp; College Station, Texas) was used for all statistical analyses.

\section{RESULTS}

\section{Patient Characteristics}

Forty-one patients underwent major amputation following a previous contralateral major amputation at our institution during the defined study period and were con- sidered for analyses. One patient who underwent a hip disarticulation was excluded and results for the remaining 40 patients are reported here. Of these, one patient was female, while the remaining patients were male (98\%). The median age of this group was $64.5 \mathrm{yr}$, with a range of 40-86 yr. Regarding age at first major lower-limb amputation, $24(60 \%)$ patients were $<65 \mathrm{yr}, 13(33 \%)$ were 66-79 $\mathrm{yr}$, and $3(8 \%)$ were $\geq 80 \mathrm{yr}$. Reasons for contralateral amputation included gangrene, osteomyelitis, nonhealing foot ulcers, and severe PAD. Most patients (30 [75\%]) had diabetes mellitus. PAD was present in $27(68 \%)$ patients in this cohort, including $20(74 \%)$ patients diagnosed with diabetes. Twenty-eight (70\%) were current smokers or had a history of cigarette smoking, and 19 (48\%) had CAD. Table 1 provides a detailed overview of comorbidities based on amputation level. A majority (22 [63\%]) were married, lived at home, and relied on a spouse for significant social support. All of the patients in this cohort were either unemployed or retired. Five patients died within 6 mo of the contralateral amputation, while 13 patients (45\%) died during follow-up. Data on inpatient rehabilitation, outpatient follow-up, and therapy were not collected for this study.

Of the 80 lower-limb amputations undergone by these 40 patients, $44(55 \%)$ were TFAs and $36(45 \%)$ were TTAs. Patients with bilateral TFAs comprised approximately half of the cohort (17 patients [43\%]) (Table 1). Patients with bilateral TTAs and patients with TFA-TTA combinations accounted for the remainder of the cohort (13 [33\%] and 10 [25\%], respectively). There were no through-knee amputations performed on patients that met inclusion/exclusion criteria. The median time

Table 1.

Baseline characteristics of Veterans with nontraumatic bilateral lower-limb loss.

\begin{tabular}{|c|c|c|c|c|}
\hline Variable & $n(\%)$ & TFA-TFA (\%) & TTA-TTA (\%) & TTA-TFA (\%) \\
\hline Male* $^{*}$ & $39(98)$ & $17(43)$ & $13(33)$ & $10(25)$ \\
\hline Diabetes Mellitus & $30(75)$ & $12(40)$ & $12(40)$ & $6(20)$ \\
\hline ESRD & $7(18)$ & $1(14)$ & $5(71)$ & $1(14)$ \\
\hline CAD & $19(48)$ & $5(26)$ & $7(37)$ & $7(37)$ \\
\hline Dementia & $2(5)$ & $0(0)$ & $2(100)$ & $0(0)$ \\
\hline Current & $9(23)$ & $4(44)$ & $3(33)$ & $2(22)$ \\
\hline Quit $>6$ mo & $19(48)$ & $8(42)$ & $6(32)$ & $5(26)$ \\
\hline No Smoking History & $9(23)$ & $4(44)$ & $3(33)$ & $2(22)$ \\
\hline
\end{tabular}

*Female $(n=1)$ : distribution reflects amputation level including female (amputation type: TTA-TFA).

$\mathrm{CAD}=$ coronary artery disease, $\mathrm{ESRD}=$ end-stage renal disease, $\mathrm{PAD}=$ peripheral arterial disease, $\mathrm{TFA}=$ transfemoral amputation, $\mathrm{TTA}=$ transtibial amputation. 
between amputations was 10 mo, with a range of 0 mo (simultaneous bilateral amputation) to $157 \mathrm{mo}$. Two patients underwent simultaneous bilateral amputation. Due to the relatively low numbers of these amputations, the decision was made to include these patients in the study. In these cases, precontralateral amputation was considered to be prebilateral amputation.

\section{Ambulation}

At baseline (i.e., with one limb intact), 19 (48\%) of the 40 patients were ambulatory. Fifteen patients (38\%) had received a limb prosthesis after the initial limb amputation, while the remaining 25 patients $(63 \%)$ had not. Most (10 [53\%]) of those who were ambulatory at baseline used an assistive device such as a cane, walker, rollator, or crutches in addition to a limb prosthesis. Of the 19 patients who were ambulatory prior to bilateral amputation, $2(11 \%)$ retained ambulatory abilities following major amputation of the contralateral limb, while the remaining 17 (89\%) became nonambulatory. Two additional patients, with bilateral TTA and TFA-TTA, who had not been ambulatory at the time of the second major amputation did ambulate after receiving a lower-limb limb prosthesis. As a result, a total of 4 patients $(10 \%$ of the overall cohort) had the ability to ambulate following bilateral lower-limb amputations.

Three patients with bilateral TTAs (23\%) and one patient with a TFA-TTA amputation (10\%) were ambulatory during follow-up (Table 2). The patient with a TFATTA reported using his right lower-limb prosthesis for transfers. Eight patients (23\% overall) received prostheses for both limbs. No patients were ambulatory following bilateral TFAs. On the whole, $15(38 \%)$ of our patients lost ambulatory capabilities, while 21 (53\%) patients lacked this capacity preoperatively. Through a Fisher exact test, a significant difference was identified between precontralateral ambulation and age categories ( $\leq 65 \mathrm{yr}, 66-79 \mathrm{yr}$, and $\geq 80 \mathrm{yr} ; p=0.049$ ). A univariate logistic regression between these two factors indicated that compared with those who were $\leq 65 \mathrm{yr}$, those between 66 and $79 \mathrm{yr}$ were 18 percent less likely to ambulate precontralateral amputation $(p=0.03)$. Other univariate analyses did not demonstrate any significant associations between postoperative ambulation and age, dementia, ESRD, or PAD $(p=0.74)$. Due to zero cells, $p$ values were not calculated for age, dementia, and ESRD. Our sample size restricted our ability to construct multivariate models identifying predictors between ambulation and potentially impactful factors.
Table 2.

Changes in ambulatory function following major amputation of second (intact) limb. Data presented as frequency (percent).

\begin{tabular}{lccc}
\hline Preoperative & \multicolumn{3}{c}{ Postoperative Ambulation } \\
\cline { 2 - 4 } TTA-TTA & Yes & No & Total \\
Yes & $2(67)$ & $5(50)$ & $7(54)$ \\
No & $1(33)$ & $5(50)$ & $6(46)$ \\
Total & $3(100)$ & $10(100)$ & $13(100)$ \\
TFA-TFA & & & \\
Yes & $0(0)$ & $6(35)$ & $6(35)$ \\
No & $0(0)$ & $11(65)$ & $11(65)$ \\
Total & $0(0)$ & $17(100)$ & $17(100)$ \\
TFA-TTA & & & \\
Yes & $0(0)$ & $6(67)$ & $6(60)$ \\
No & $1(100)$ & $3(33)$ & $4(40)$ \\
Total & $1(100)$ & $9(100)$ & $10(100)$ \\
All & & & \\
Yes & $2(50)$ & $17(53)$ & $19(48)$ \\
No & $2(50)$ & $19(47)$ & $21(53)$ \\
Total & $4(100)$ & $36(100)$ & $40(100)$ \\
\hline TFA = transfemoral amputation, TTA $=$ transtibial amputation. &
\end{tabular}

\section{Functional Independence Measure and Medicare K-Levels}

Pre and post FIMs were available in 23 (58\%) patients. Following amputation of the second limb, FIM scores decreased in 17 patients $(74 \%)$, increased in 5 patients $(22 \%)$, and remained unchanged in 2 patients (9\%). The median FIM score change was 11 points following amputation of the second limb. More decreasing scores, by frequency, were observed in patients with TTA-TTA combinations than in TFA-TFA or TFA-TTA combinations $(30 \%$ vs $26 \%$ vs $17 \%$, respectively; $p=$ 0.59 ) but the observed differences were not significant.

Data from the Medicare K-Level scale was assessed to further illuminate patient ambulatory capabilities. KLevel classifications were available in 31 patients $(78 \%)$, with 5 patients (16\%) classified at level 0 prior to contralateral amputation. Postcontralateral amputation, this percentage increased to 19 (6 patients). No patients were classified at K-Level 4, while $2(6 \%)$ were classified level 3 at unilateral amputation. Postcontralateral amputation, all patients were classified Levels 0,1 , or 2.

\section{Independence}

Before undergoing major amputation of the second (contralateral) limb, 35 (88\%) patients were independent 
and lived at home. Five patients (13\%) lived in a nursing home or other care facility. After amputation of the second (contralateral) limb, only 21 (53\%) remained independently living. While no significant association was seen between loss of independence and specific level of amputation, 6 of the 14 patients $(43 \%)$ who lost independence had undergone bilateral TFAs and $5(26 \%)$ of all those who were nonindependent lived alone prior to contralateral amputation (Table 3). Overall, 8 patients $(20 \%)$ were either residing in a skilled nursing facility or nursing home after bilateral amputation. There was a significant difference in groups between preoperative independent living and postoperative independent living $(p=0.02)$ as well as preoperative independent living and the use/issuance of a prosthesis $(p=$ 0.056). Details of the magnitude and direction of this difference could not be further elucidated through univariate analyses. No significant associations were found between postcontralateral amputation independence and age $(p=$ $0.99)$, dementia $(p=0.51)$, ESRD $(p=0.58)$, amputation type $($ reference $=$ TTA-TTA, TFA-TFA: $p=0.13$, TTATFA: $p=0.35)$, or baseline ambulatory function $(p=0.99)$.

\section{DISCUSSION}

In this sample of Veteran patients, with high rates of comorbid conditions, a large number of patients had previous attempts at revascularization and multiple minor lower-limb amputations. Given the presence of serious coexisting conditions, it is important to note that more than half the cohort (53\%) was already nonambulatory prior to contralateral amputation. While two patients from this fraction became ambulatory after contralateral amputation, there is no discounting the physiological and psychological toll that losing both limbs has on a patient of advanced years. Thus, it is not surprising that the results of this study suggest even poorer functional outcomes among a largely dysvascular Veteran population than had been previously reported. Specifically, only 11 percent of Veterans in our cohort remained ambulatory after undergoing bilateral major amputations - much lower than the 38 percent rate reported by Inderbitzi et al. [6]. With a similarly comorbid study population of 66 patients, Inderbitzi et al. reported the mortality rate at 69 percent after $5 \mathrm{yr}$ and coronary heart disease the leading cause of death [6].

In general, why might the outcomes observed in our cohort be so poor? It may be due to the fact that the number of patients within the Veterans Health Administration
Table 3.

Changes in independent living status function following major amputation of second (intact) limb. Data presented as frequency (percent).

\begin{tabular}{lccc}
\hline Preoperative & \multicolumn{3}{c}{ Postoperative Independence } \\
\cline { 2 - 4 } Independence & Yes & No & Total \\
\hline Yes & $9(100)$ & $3(75)$ & $12(92)$ \\
No & $0(0)$ & $1(25)$ & $1(8)$ \\
Total & $9(100)$ & $4(100)$ & $13(100)$ \\
TFA-TFA & & & \\
Yes & $7(100)$ & $6(60)$ & $13(76)$ \\
No & $0(0)$ & $4(40)$ & $4(24)$ \\
Total & $7(100)$ & $10(100)$ & $17(100)$ \\
TFA-TTA & & & \\
Yes & $5(100)$ & $5(100)$ & $10(100)$ \\
No & $0(0)$ & $0(0)$ & $0(0)$ \\
Total & $5(100)$ & $5(100)$ & $10(100)$ \\
All & & & \\
Yes & $21(100)$ & $14(74)$ & $35(88)$ \\
No & $0(0)$ & $5(26)$ & $5(13)$ \\
Total & $21(100)$ & $19(100)$ & $40(100)$ \\
\hline TFA = transfemoral amputation, TTA = transtibial amputation. &
\end{tabular}

with diabetes is nearly twice that of the general population within the United States (20\% vs 9\%, respectively) and the proportion that are elderly is nearly three times that of the general population ( $37 \%$ vs $13 \%$, respectively) [15-17]. Additionally, Veterans with limb loss are known to have low degrees of social support, low income, and negative health behaviors that heighten the risk of limb loss [18-20]. Indeed, it may be true that the rates here are representative of the elderly predominately dysvascular patient population seen at many large VA medical centers and many large, urban non-Federal medical centers in the United States.

As the more comprehensive of the two measures, the FIM was more attuned to specific changes in the functional capabilities of a patient than the Medicare K-Levels. The broad categories of the K-Levels, arguably, allow for more subjective misclassification than the FIM scores. Nevertheless, both measurement tools showed that functional capabilities declined in this cohort. Interestingly, decreasing FIM scores were observed more often in TTATTA patients than in the other groups. This might be due to the absence of scores in the other amputation categories leading to an appearance of greater success in that group. The ability to live independently declined slightly after 
contralateral amputation. Confirming intuitive reasoning, there seems to be a possible association between preoperative independent living and postoperative independent living. Also, the issuance or use of a prosthesis might have an association with preoperative independent living. Due to the nature of our sample, we were unable to further expound on whether these associations were protective or harmful.

As with any retrospective chart review analysis, our study was dependent on the accuracy and frequency of information recorded in the patient's chart. In some instances, a patient's self-reported ambulatory capability varied between visits and examinations. Additionally, the lack of power due to a relatively small sample size weakened our statistical analysis. Some aspects of our analysis shed light on statistically significant factors of interest (i.e., indication that significant differences were present between some groups and the lessened likelihood of ambulation with unilateral ambulation in 66-79 $\mathrm{yr}$ olds). However, the regressions should be interpreted with caution because in all cases the models were univariate and were not adjusted for confounding factors. While the presence of differences between groups could be confirmed statistically in some cases, confirmation of the direction and magnitude of these differences was unavailable. We were limited by small cell sizes and, therefore, cannot definitively discount the lack of significant association between postoperative independence and postoperative ambulation against some of the other independent variables. Yet, our results provide insights into areas that merit further investigation using a wellpowered sample size to more effectively elucidate significant associations.

The poorer clinical and functional outcomes seen in patients with a history of unilateral lower-limb amputation should not dissuade clinicians from offering revascularization and aggressive limb preservation efforts to this patient population. Indeed, the dismal functional outcomes (based on the percentage of patients who lost ambulatory capabilities - 90\%) reported in the current study should provide a strong argument for limb salvage attempts. The decision to offer revascularization to ambulatory and/or independent patients with previous unilateral lower-limb amputation should be at least as aggressive as it is in patients without such a history. In essence, it is important to have an honest discussion regarding the costs and benefits of amputation versus revascularization supported by evidence from the litera- ture. Moreover, the collaboration of an interdisciplinary team in the care of patients at risk for bilateral amputations can provide valuable perspective and encourage long-term gains in the delivery of quality healthcare.

\section{CONCLUSIONS}

Functional outcomes and independence were largely poor in this sample of dysvascular Veteran patients with nontraumatic bilateral lower-limb amputations. Similar to other studies and based on the number of patients ambulatory in each amputation category [18-19], the current study allows for the inference that the preservation of at least one knee joint has a significant effect on ambulation and the ability to live independently. Further studies with larger sample sizes are needed to more robustly identify factors associated with a loss of ambulatory ability and/or a loss of the ability to live independently following major amputation.

\section{ACKNOWLEDGMENTS}

\section{Author Contributions:}

Study concept and design: N. R. Barshes.

Acquisition of data: S. E. Sharath

Analysis and interpretation of data: S. E. Sharath, N. R. Barshes.

Drafting of manuscript: S. E. Sharath, N. R. Barshes.

Critical revision of manuscript for important intellectual content:

S. E. Sharath, H. Henson, S. Flynn, G. Pisimisis, P. Kougias,

N. R. Barshes.

Statistical analysis: S. E. Sharath, N. R. Barshes.

Study supervision: N. R. Barshes.

Financial Disclosures: The authors have declared that no competing interests exist.

Funding/Support: This material was unfunded at the time of manuscript preparation.

Institutional Review: This study was approved by the Baylor College of Medicine Institutional Review Board (protocol H-32460).

Approval was also granted by the VA Research and Development Committee. As a retrospective database study, the institutional review board determined that a waiver of informed consent was appropriate for this research.

Participant Follow-Up: The authors have no plans to notify the study subjects of the publication of this article because of a lack of contact information. 


\section{REFERENCES}

1. Baril DT, Goodney PP, Robinson WP, Nolan BW, Stone DH, Li Y, Cronenwett JL, Schanzer A; Vascular Study Group of New England. Prior contralateral amputation predicts worse outcomes for lower extremity bypasses performed in the intact limb. J Vasc Surg. 2012;56(2):353-60. [PMID:22480762]

http://dx.doi.org/10.1016/j.jvs.2012.01.041

2. Abou-Zamzam AM Jr, Lee RW, Moneta GL, Taylor LM Jr, Porter JM. Functional outcome after infrainguinal bypass for limb salvage. J Vasc Surg. 1997;25(2):287-95, discussion 295-97. [PMID:9052563] http://dx.doi.org/10.1016/S0741-5214(97)70350-1

3. Goodney PP, Likosky DS, Cronenwett JL; Vascular Study Group of Northern New England. Predicting ambulation status one year after lower extremity bypass. J Vasc Surg. 2009;49(6):1431-39.e1. [PMID:19497502] http://dx.doi.org/10.1016/j.jvs.2009.02.014

4. Suckow BD, Goodney PP, Cambria RA, Bertges DJ, Eldrup-Jorgensen J, Indes JE, Schanzer A, Stone DH, Kraiss LW, Cronenwett JL; Vascular Study Group of New England. Predicting functional status following amputation after lower extremity bypass. Ann Vasc Surg. 2012;26(1): 67-78. [PMID:22176876]

http://dx.doi.org/10.1016/j.avsg.2011.07.014

5. Barshes NR, Belkin M; MOVIE Study Collaborators. A framework for the evaluation of "value" and costeffectiveness in the management of critical limb ischemia. J Am Coll Surg. 2011;213(4):552-66.e5. [PMID:21943802] http://dx.doi.org/10.1016/j.jamcollsurg.2011.07.011

6. Inderbitzi R, Buettiker M, Enzler M. The long-term mobility and mortality of patients with peripheral arterial disease following bilateral amputation. Eur J Vasc Endovasc Surg. 2003;26(1):59-64. [PMID:12819649] http://dx.doi.org/10.1053/ejvs.2002.1868

7. Lavery LA, Van Houtum WH, Armstrong DG. Institutionalization following diabetes-related lower extremity amputation. Am J Med. 1997;103(5):383-88. [PMID:9375706] http://dx.doi.org/10.1016/S0002-9343(97)00163-0

8. Faglia E, Clerici G, Mantero M, Caminiti M, Quarantiello A, Curci V, Morabito A. Incidence of critical limb ischemia and amputation outcome in contralateral limb in diabetic patients hospitalized for unilateral critical limb ischemia during 1999-2003 and followed-up until 2005. Diabetes Res Clin Pract. 2007;77(3):445-50. [PMID:17316866] http://dx.doi.org/10.1016/j.diabres.2007.01.010

9. Johannesson A, Larsson GU, Ramstrand N, Turkiewicz A, Wiréhn AB, Atroshi I. Incidence of lower-limb amputation in the diabetic and nondiabetic general population: A 10year population-based cohort study of initial unilateral and contralateral amputations and reamputations. Diabetes Care. 2009;32(2):275-80. [PMID:19001192]

http://dx.doi.org/10.2337/dc08-1639

10. Castro MP, Soares D, Mendes E, Machado L. Center of pressure analysis during gait of elderly adult transfemoral amputees. J Prosthet Orthot. 2013;25:68-75. http://dx.doi.org/10.1097/JPO.0b013e31828c04b0

11. Barshes NR, Chambers JD, Cohen J, Belkin M; Model To Optimize Healthcare Value in Ischemic Extremities 1 (MOVIE) Study Collaborators. Cost-effectiveness in the contemporary management of critical limb ischemia with tissue loss. J Vasc Surg. 2012;56(4):1015-24.e1.

[PMID:22854267]

http://dx.doi.org/10.1016/j.jvs.2012.02.069

12. Dodds TA, Martin DP, Stolov WC, Deyo RA. A validation of the Functional Independence Measurement and its performance among rehabilitation inpatients. Arch Phys Med Rehabil. 1993;74(5):531-36. [PMID:8489365] http://dx.doi.org/10.1016/0003-9993(93)90119-U

13. Ottenbacher KJ, Hsu Y, Granger CV, Fiedler RC. The reliability of the Functional Independence Measure: A quantitative review. Arch Phys Med Rehabil. 1996;77(12):122632. [PMID:8976303] http://dx.doi.org/10.1016/S0003-9993(96)90184-7

14. The American Academy of Orthotists and Prosthetists. Outcome measures in lower limb prosthetics: K-Levels [Internet]. Washington (DC): The American Academy of Orthotists and Prosthetists; [cited 2015 Jan]. Available from: http://www.oandp.org/olc/ course extended content.asp?frmCourseId $=\mathrm{ACA}$ 066EC443A-4822-822C-89BC1CBD684E\& frmTermId=k-levels

15. Miller DR, Safford MM, Pogach LM. Who has diabetes? Best estimates of diabetes prevalence in the Department of Veterans Affairs based on computerized patient data. Diabetes Care. 2004;27(Suppl 2):B10-21. [PMID:15113777] http://dx.doi.org/10.2337/diacare.27.suppl_2.B10

16. National Center for Veterans Analysis and Statistics. Profile of Veterans 2009: Data from the American Community Survey. Washington (DC): National Center for Veterans Analysis and Statistics; 2011.

17. U.S. Census Bureau. Age and sex-The older population in the United States: 2010. Suitland (MD): U.S. Census Bureau; 2012 Nov [cited 2012 Nov]. Available from: http://www.census.gov/population/age/data/2010.html

18. Piette JD, Kerr E, Richardson C, Heisler M. Veterans Affairs research on health information technologies for diabetes self-management support. J Diabetes Sci Technol. 2008;2(1):15-23. [PMID:19885173] http://dx.doi.org/10.1177/193229680800200104

19. Czerniecki JM, Turner AP, Williams RM, Hakimi KN, Norvell DC. The effect of rehabilitation in a comprehensive inpatient rehabilitation unit on mobility outcome after 
dysvascular lower extremity amputation. Arch Phys Med Rehabil. 2012;93(8):1384-91. [PMID:22465582]

http://dx.doi.org/10.1016/j.apmr.2012.03.019

20. Olson JM, Hogan MT, Pogach LM, Rajan M, Raugi GJ, Reiber GE. Foot care education and self management behaviors in diverse veterans with diabetes. Patient Prefer Adherence. 2009;3:45-50. [PMID:19936144]

http://dx.doi.org/10.2147/PPA.S4349

Submitted for publication July 31, 2014. Accepted in revised form June 17, 2015.

This article and any supplementary material should be cited as follows:
Sharath S, Henson H, Flynn S, Pisimisis G, Kougias P, Barshes NR. Ambulation and independence among Veterans with nontraumatic bilateral lower-limb loss. J Rehabil Res Dev. 2015;52(7):851-58.

http://dx.doi.org/10.1682/JRRD.2014.07.0176

ORCID: Sherene E. Sharath, MPH: 0000-0001-53345799; Panos Kougias, MD, MS: 0000-0001-8561-2185; Neal R. Barshes, MD, MPH: 0000-0003-1019-8930

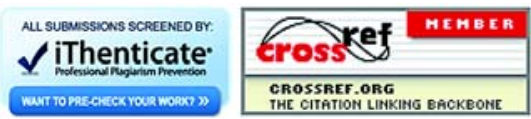

